\title{
Impact of belimumab on patient-reported outcomes in systemic lupus erythematosus: review of clinical studies
}

This article was published in the following Dove Medical Press journal:

Patient Related Outcome Measures

\author{
Elvira Bangert ${ }^{\prime}$ \\ Laura Wakani² \\ Mehveen Merchant ${ }^{3}$ \\ Vibeke Strand ${ }^{4}$ \\ Zahi Touma ${ }^{2}$ \\ 'Division of Rheumatology, \\ Department of Medicine, Queen's \\ University, Kingston, ON, Canada; \\ ${ }^{2}$ University of Toronto Lupus \\ Clinic, Toronto Western Hospital, \\ Centre for Prognosis Studies in the \\ Rheumatic Diseases, Toronto, ON, \\ Canada; ${ }^{3}$ Division of Rheumatology, \\ Nova Scotia Health Authority, \\ Dalhousie University, Halifax, NS, \\ Canada; ${ }^{4}$ Division of Immunology/ \\ Rheumatology, Stanford University, \\ Palo Alto, CA, USA
}

Correspondence: Zahi Touma

University of Toronto Lupus Clinic,

Toronto Western Hospital, Centre

for Prognosis Studies in the Rheumatic

Diseases, 399 Bathurst Street,

Toronto, ON, Canada

Tel + I 4166035800 ext 5743

Fax + I 4166039387

Email Zahi.Touma@uhn.ca

\begin{abstract}
Systemic lupus erythematosus (SLE) is a chronic autoimmune, multisystem rheumatic disease with significant impact on health-related quality of life (HRQoL). Patientreported outcomes (PROs) provide valuable data on patient perceptions across a variety of domains, such as HRQoL, pain, fatigue, and depression. The measurement and results of PROs with respect to HRQoL in randomized controlled trials (RCTs) on belimumab (B-lymphocyte stimulator inhibitor) in SLE are reviewed here, including BLISS-52 and BLISS-76, as well as publications related to belimumab trials that included HRQoL data. Other trials that evaluated belimumab did not include HRQoL data and were therefore not included in the analysis. The BLISS-52 and BLISS-76 RCTs met their primary endpoints and demonstrated improvements in PROs, measured by the 36-item Short Form Health Survey, EuroQol 5 Dimensions, and Functional Assessment of Chronic Illness Therapy-Fatigue Scale. Belimumab was shown overall to improve PROs in adult autoantibody-positive lupus patients.
\end{abstract}

Keywords: quality of life, fatigue, systemic lupus, Benlysta, trials

\section{Introduction}

Systemic lupus erythematosus (SLE) is a chronic autoimmune, multisystem rheumatic disease with significant impact on many dimensions of patients' well-being, causing marked impairment in health-related quality of life (HRQoL). ${ }^{1}$ Evidence suggests that the impact on HRQoL experienced by SLE patients is not adequately captured by assessments of physician-assessed disease activity and damage alone. ${ }^{2}$ Patientreported outcomes (PROs) reflect the patient's perspectives of their illness, HRQoL, and well-being, and can be helpful in informing treatment decisions. ${ }^{1}$ Recognizing their value, PROs are identified as an independent core domain by the Outcome Measures in Rheumatology (OMERACT) and other regulatory agencies and health care decision makers. OMERACT recommended four domains to be assessed in SLE randomized controlled trials (RCTs) and longitudinal observational studies: disease activity, HRQoL, damage, and adverse events. ${ }^{3}$ The US Food and Drug Administration (FDA) requires that control group participants in SLE RCTs receive, at minimum, standard of care therapy. ${ }^{4}$ Detecting a difference in endpoints between the treatment and control groups may be challenging if a similar level of response to the experimental, add-on treatment is observed. To address this challenge, responder vs non-responder analysis is used to detect differences in outcomes. The metrics and results of PROs in the belimumab RCTs in SLE are reviewed here. 


\section{Belimumab in SLE}

Similar to the clinical heterogeneity of SLE, ${ }^{5}$ its etiopathogenesis is also multifactorial, involving environmental (eg, sex hormones, ultraviolet light, viral infections), genetic and epigenetic, hormonal, and immunologic factors. ${ }^{6,7}$ In genetically predisposed individuals, a normal immune response is impaired, leading to increased autoantibodies and activation of the innate and adaptive immune responses. ${ }^{6,89}$ Patients with SLE have both abnormal activation pathways and activation of a large proportion of $\mathrm{B}$ and $\mathrm{T}$ cells causing autoimmunity and inflammation. ${ }^{8} \mathrm{~B}$ cells play a crucial role in the pathogenesis of SLE, including acting as precursors for plasma cells that secrete autoantibodies, presenting antigenic peptides to $T$ cells, and secreting pro-inflammatory cytokines that amplify and downregulate immune responses. ${ }^{8-12}$ Not surprisingly, given the central role for B cells in the pathogenesis of SLE, B cells emerged as a potential therapeutic target. ${ }^{13}$ Recent therapeutic interventions focus on strategies such as inhibiting B-cell activation, decreasing B-cell survival, or deleting specific lineages of $\mathrm{B}$ cells. ${ }^{10} \mathrm{~B}$-lymphocyte stimulator (BLyS), also known as B-cell activating factor (BAFF), and a proliferation-inducing ligand, are cytokines belonging to the tumor necrosis factor ligand superfamily; they activate B-cell stimulation and maturation of B cells through the binding of several receptors on B cells. ${ }^{14,15}$ Studies have demonstrated that BLyS levels are correlated with anti-dsDNA antibody levels and SLE disease activity, ${ }^{14-16}$ therefore representing a potentially important therapeutic target in SLE. ${ }^{13}$ Belimumab (Benlysta) is a human IgG1k monoclonal antibody that binds BLyS/BAFF, thus preventing it from binding to its receptors on B cells. Consequently, belimumab depletes activated and naïve $\mathrm{B}$ cells but not directly memory B cells. ${ }^{8,14,17}$ In 2011, intravenous (IV) belimumab was approved for the treatment of SLE, the first time for a new therapy in $>50$ years. ${ }^{13}$ In 2017 , the subcutaneous belimumab formulation was approved in the USA, Japan, and the European Union. ${ }^{18,19}$

\section{RCTs of belimumab in SLE}

The efficacy of belimumab in addition to standard of care in adult patients with autoantibody-positive SLE has been demonstrated in four phase III, multicenter, prospective RCTs. (Table 1 summarizes the trials.) Inclusion criteria for the four trials were very similar and differences are highlighted in Table 1: age $\geq 18$ years, classification of SLE according to the American College of Rheumatology criteria, seropositivity for autoantibodies, and a stable treatment regimen with glucocorticosteroids, antimalarials, or immunosuppressives for at least 30 days before the first study dose. In concordance with OMERACT recommendations and regulatory agencies, the belimumab trials assessed the robustness of responses across four domains: disease activity, damage, adverse effects, and HRQoL. ${ }^{3,20-23}$ The primary outcome measure in all four RCTs was the Systemic Lupus Erythematosus Responder Index-4 (SRI-4) at week 52. The RCTs demonstrated significant improvement in the SRI-4 with $10 \mathrm{mg} / \mathrm{kg}$ IV belimumab compared with placebo. ${ }^{20-22}$ Improvements in multiple domains were reported vs placebo and the 10 $\mathrm{mg} / \mathrm{kg}$ dose received formal approval for treatment of SLE by the US FDA, Health Canada, and European Medicines

Table I Summary of the trials

\begin{tabular}{|c|c|c|c|c|}
\hline RCT & BLISS-52 ${ }^{20}$ & BLISS-76 ${ }^{21}$ & $\begin{array}{l}\text { Northeast Asia } \\
\text { Trial }^{22}\end{array}$ & BLISS-SC $^{23}$ \\
\hline Duration (weeks) & 52 & 76 & 52 & 52 \\
\hline Number of patients & 852 & 819 & 677 & 836 \\
\hline Location & $\begin{array}{l}\text { Eastern Europe, Asia-Pacific, } \\
\text { and Latin America }\end{array}$ & $\begin{array}{l}\text { North and Central } \\
\text { America, and Europe }\end{array}$ & $\begin{array}{l}\text { China, Japan, and South } \\
\text { Korea }\end{array}$ & $\begin{array}{l}\text { North, Central, and South } \\
\text { America, Eastern and } \\
\text { Western Europe, Australia } \\
\text { and Asia }\end{array}$ \\
\hline $\begin{array}{l}\text { Belimumab form of } \\
\text { administration }\end{array}$ & Intravenous & Intravenous & Intravenous & Subcutaneous \\
\hline $\begin{array}{l}\text { Belimumab dose } \\
\text { groups }\end{array}$ & $\begin{array}{l}\mathrm{I} \mathrm{mg} / \mathrm{kg} \text { or } 10 \mathrm{mg} / \mathrm{kg} \text { days } 0 \text {, } \\
\mathrm{I} 4 \text {, and } 28 \text {, and then every } \\
28 \text { days until } 48 \text { weeks }\end{array}$ & $\begin{array}{l}\mathrm{I} \mathrm{mg} / \mathrm{kg} \text { or } 10 \mathrm{mg} / \mathrm{kg} \text { days } \\
0,14 \text {, and } 28 \text {, and every } \\
28 \text { days through week } 72\end{array}$ & $\begin{array}{l}10 \mathrm{mg} / \mathrm{kg} \text { on days } 0,14, \\
\text { and } 28 \text {, and then every } \\
28 \text { days up to week } 48\end{array}$ & 200 mg weekly \\
\hline $\begin{array}{l}\text { SELENA-SLEDAI } \\
\text { scores for inclusion }\end{array}$ & $\geq 6$ & $\geq 6$ & $\geq 8$ & $\geq 8$ \\
\hline
\end{tabular}

Abbreviations: BLISS-52, Study of Belimumab in Subjects with SLE 52-week trial; BLISS-76, Study of Belimumab in Subjects with SLE 76-week trial; BLISS-SC, Study of Belimumab Administered Subcutaneously in Subjects with SLE; RCT, randomized controlled trial; SELENA-SLEDAI, Safety of Estrogens in Lupus Erythematosus National Assessment-SLE Disease Activity Index. 
Agency. ${ }^{25,26}$ In the pooled data analyses of the BLISS-52 and BLISS-76, responses occurred in $50.6 \%$ of patients assigned to belimumab $10 \mathrm{mg} / \mathrm{kg}$ vs $38.6 \%$ placebo $(P<0.001) .{ }^{27}$ Across the pooled data of the BLISS-52 and BLISS-76 trials, belimumab $10 \mathrm{mg} / \mathrm{kg}$ was associated with improvements in multiple domains, including: disease activity, demonstrated by reductions from baseline in Safety of Estrogens in Lupus Erythematosus National Assessment-SLE Disease Activity Index scores at week 52; reductions in the incidence and severity of flares; and decreased glucocorticosteroid doses. ${ }^{28}$ The BLISS-SC trial demonstrated similar treatment effects to the $10 \mathrm{mg} / \mathrm{kg}$ IV doses in BLISS-52 and BLISS-76. Importantly, patients with severe active lupus nephritis or central nervous system lupus were excluded from all four trials. During post-marketing experience, in an open-label extension over a 7-year period (1,746 patient-years), adverse events rates due to belimumab either remained stable or substantially decreased. ${ }^{29}$

\section{PROs in the belimumab trials}

Across all trials, there was an overall consensus that belimumab was superior to placebo, reducing the burden of disease with a safe medication profile.

Besides physician-centered indices, the BLISS-52 and BLISS-76 trials (but not the Northeast Asia Trial) evaluated PROs relevant to HRQoL in SLE, including the generic Medical Outcomes Study 36-item Short Form Health Survey (SF-36 v2); secondary analyses were also performed using other generic questionnaires: the Functional Assessment of Chronic Illness Therapy-Fatigue Scale (FACIT-F) and the EuroQol 5 Dimensions (EQ-5D). ${ }^{30,31}$ Although measuring multiple aspects of HRQoL, these instruments together capture two of the most frequently reported symptoms in SLE: fatigue and pain. ${ }^{32}$ More specifically, all three - SF-36, FACIT-F, and EQ-5D - capture fatigue, and SF-36 and EQ-5D capture pain as well. As secondary endpoints, these two trials included mean changes in the SF-36 Physical Component Summary (PCS) and Mental Component Summary (MCS) scores (at weeks 24 and 52 for BLISS-52, and weeks 24, 52, and 76 for BLISS-76); FACIT-F, and EQ-5D scores at various time points (weeks 12, 24, 52, and 76) (BLISS-76 only).$^{30}$ Analyses of PRO data from both the BLISS-52 and BLISS-76 trials were performed according to SRI-4 responders vs non-responders across all doses by SF-36 summary and domain scores, FACIT-F and EQ-5D (at weeks 12, 24, and 52, and, for BLISS-76 data, also at 76 weeks). ${ }^{30}$ In the BLISS-SC trial, FACIT-F was administered at weeks 4, 8, 12,
24,36 , and 52 and analyzed with the corresponding visits. ${ }^{23}$ The PROs used to measure HRQoL and fatigue, and their results from the BLISS trials, will be reviewed here.

\section{6-Item Short Form Health Survey}

The SF-36 was first developed in 1988 and was followed by the Standard SF-36 Health Survey in 1992, containing 36 questions, and is one of the most widely used and studied generic PRO questionnaires in SLE. ${ }^{33}$ It measures various domains from the WHO quality of life list of domains. ${ }^{2}$ The questionnaire is scored from 0 (worse health) to 100 (better health), capturing eight domains: physical functioning, role physical, bodily pain, general health, vitality, social functioning, role emotional, and mental health. ${ }^{24}$ From these domains, the PCS and MCS are calculated. ${ }^{24,34}$ Since the instrument has been introduced in the late 1980s, its psychometric measurement properties across cultures and languages have been well established and it continues to be one of the most common questionnaires included in clinical research studies and RCTs. ${ }^{1,35-38}$ The reliability (internal consistency ${ }^{39,40}$ and test-retest reliability ${ }^{39-41}$ ), validity ${ }^{42-46}$ and responsiveness ${ }^{42,44,45,47}$ of SF-36 have been extensively studied.

It is important to note that PRO measures, such as the SF-36, are to be interpreted in the context of minimal clinically important differences (MCIDs). MCIDs are an important concept used to determine whether a medical intervention improves outcomes perceived by patients to be clinically meaningful. ${ }^{37,48}$ A clinical intervention is considered clinically meaningful if that PRO score change at follow-up meets or exceeds the MCID for that instrument in that disease. ${ }^{38}$ MCIDs for improvement and worsening in SF-36 have been established in SLE, as well as MCIDs for FACIT: $\geq 2.5$ point increases and $\geq-0.8$ decreases in PCS and MCS scores for improvement and worsening, respectively, $\geq 5.0$ point increases and $\geq-2.5$ decreases in SF-36 individual domain scores for improvement and worsening, respectively, ${ }^{49,50}$ and $\geq 4.0$ MCID for FACIT improvement; and were applied to the analyses of SF-36 in the BLISS trials. Mean SF-36 domain scores in patients at baseline and week 52 were compared with age- and gender-matched healthy US subjects. ${ }^{20,21}$ The well-established MCID definition of improvements of $\geq 10$ points on a $0-100$ VAS was used for EQ-5D.

A combined analysis across both RCTs revealed that at week 52 mean improvements in SF-36 PCS scores were greater in SRI-4 responders vs non-responders (4.9 vs 2.6; $P<0.001)$ and exceeded MCID. ${ }^{31}$ Similarly, at week 52, mean improvements in SF-36 MCS were greater in SRI-4 
responders vs non-responders (4.4 vs $1.7 ; P<0.001)$, and also exceeded MCID. ${ }^{31}$ In addition, all SF-36 domain scores showed statistically significant improvements in responders vs non-responders $(P<0.001) .^{31}$

Further post-hoc analysis was performed. At 24 weeks, the first major prespecified endpoint of both BLISS trials demonstrated that mean improvements from baseline in SF-36 PCS did not differ significantly between treatment arms with belimumab $10 \mathrm{mg} / \mathrm{kg}$ and placebo $(+3.34 \mathrm{vs}+3.26)$ in BLISS-52 and $(+3.21$ vs +3.35$)$ in BLISS-76. ${ }^{20,21}$ However, by week 52, significant improvements were reported in BLISS-52 PCS scores (+4.19 vs $+2.84 ; P=0.0247)$ but not in BLISS-76 $(+3.44 \mathrm{vs}+2.85) .{ }^{20,21} \mathrm{In}$ BLISS-52, improvements were noted with the $1 \mathrm{mg} / \mathrm{kg}$ and 10 $\mathrm{mg} / \mathrm{kg}$ belimumab doses in the following SF-36 domains at 52 weeks: physical functioning, bodily pain, and role-emotional. With the $1 \mathrm{mg} / \mathrm{kg}$ dose, there were improvements in the social functioning and general health domains as well. In BLISS-76, statistically significant improvements were reported with the $1 \mathrm{mg} / \mathrm{kg}$ dose in the following domains: role-physical, bodily pain, general health, and vitality at week 52 .

\section{Functional Assessment of Chronic Illness Therapy-Fatigue Scale}

The FACIT-F is a 13-item fatigue questionnaire that assesses self-reported aspects of physical and mental fatigue and impact on function and daily living. ${ }^{2,51}$ Items are scored using a four-point Likert scale. The score ranges from 0 to 52 and lower scores represent more fatigue. The FACIT-F was originally developed in $1997^{52}$ for the assessment of fatigue in patients with anemia, and validated for use in rheumatic diseases. ${ }^{53}$ The content validity of the FACIT-F was confirmed by Kosinski et $\mathrm{al}^{54}$ in lupus patients. Other psychometric properties of FACIT-F, reliability, ${ }^{55}$ validity, ${ }^{30,51,55}$ and responsiveness ${ }^{31}$ have been demonstrated in several trials. In the SLE population, the instrument's ability to detect change over time has been consistently demonstrated. ${ }^{2}$

Similar to the outcomes of the SF-36 in the BLISS trials, at week 52, SRI-4 responders reported higher mean improvements in FACIT-F scores (5.2) compared to non-responders (3.0), but only values reported by SRI-4 responders exceeded the MCID. ${ }^{31}$ Both belimumab treatment groups demonstrated significant improvements by week 52 compared to placebo, with greater improvements by week 8 in responders, which were sustained through week 52. ${ }^{31}$ In post-hoc subgroup analyses of FACIT-F in Hispanic or Latino patients in the BLISS-SC trial, improvements were reported over time in both treatment groups and mean changes from baseline were significantly greater in the belimumab compared with placebo groups at weeks 8,36 , and 52 , but not weeks 4,12 , and 24 . At week 52, more patients who received belimumab reported improvements that equaled or exceeded the MCID in FACIT-F scores $\geq 4$ compared with placebo ( $44.4 \%$ vs $36.1 \%$; OR 1.42 , 95\% CI 1.05-1.94; $P=0.0245){ }^{23}$

\section{EuroQol 5 Dimensions}

The EQ-5D is composed of a six-item questionnaire and a VAS, allowing patients to rate their overall health. The VAS is scored from 0 to 100 , reflecting "worst imaginable health" to "best imaginable health". The EQ-5D VAS score permits an economic evaluation, through the calculation of qualityadjusted life-years. The questionnaire portion is a three-item response scale, reporting results as a summary score from 0 to 1 , reflecting "death or health worse than death" to "best imaginable health". ${ }^{1}$ The impact on disability is assessed in the following five domains: mobility, self-care, usual activities, pain/discomfort, and anxiety/depression. ${ }^{1,2}$ The reliability and validity of the EQ-5D have been established in the SLE population and it is commonly used in RCTs. ${ }^{2}$ The EQ-5D was evaluated in the BLISS-52 and BLISS-76 RCTs, with changes in the pain/discomfort domain noted. ${ }^{30}$

\section{Discussion}

In the phase III BLISS RCTs, the primary endpoints were met and a combined analysis of SRI-4 responders vs non responders across all treatment groups demonstrated statistically significant and clinically meaningful improvements in PROs. ${ }^{31}$ These findings supported the approval of belimumab for treatment of patients with adult autoantibody-positive SLE.

As per OMERACT recommendations, both generic and disease-specific instruments should be used to gauge HRQoL; disease-specific PRO instruments can complement generic ones such as SF-36. ${ }^{56,57}$ While generic instruments allow comparison to other diseases, disease-specific ones may contribute additional information. ${ }^{57}$ The ultimate goal with these PROs is to identify areas that could potentially be targeted with therapeutic interventions. ${ }^{57}$

Generic PRO questionnaires frequently evaluated in SLE include the Medical Outcomes Study SF-36, EQ-5D, and the FACIT-F. Several HRQoL domains of importance to SLE patients, such as appearance, confidence, and body image, are not represented in the generic PRO tools such as SF-36. SLE-specific PRO instruments have been developed to capture these domains. These SLE-specific tools include the LupusQoL, SLE Symptom Checklist (SSC), SLE Quality of Life (SLEQoL), Lupus Quality of Life (L-QoL), Lupus Patient-Reported Outcome tool (LupusPRO), Lupus Impact 
Tracker (LIT), Simple Measure of Impact of Lupus Erythematosus in Youngsters (SMILEY), and Body Image in Lupus Scale (BILS). ${ }^{1,2}$

In SLE, pain, fatigue, and physical function are frequently assessed both by generic PROs, such as the SF-36 and EQ-5D, and by the SLE-specific LupusQoL and LupusPRO questionnaires. ${ }^{2}$ The review by Holloway et al in 2014 showed that the SF-36 v2, the LupusQoL, and the FACIT-F demonstrated content and face validity and overall strong psychometric properties in an SLE population. ${ }^{32}$ More recently, in 2018 , Izadi et al reviewed the literature to assess the responsiveness of different PROs to interventions in RCTs. This review confirmed that SF-36 and LupusQoL have strong evidence for adequate psychometric measurement properties. ${ }^{58}$ In addition, the LIT and Patient-Reported Outcomes Measurement Information System (PROMIS) showed strong evidence for measurement properties; however, PROMIS responsiveness has not been studied in lupus, nor have LupusPRO or LIT been assessed in RCTs.

LupusQoL is an SLE-specific HRQoL capturing the following eight HRQoL domains: physical health, emotional health, body image, pain, planning, fatigue, intimate relationships, and burden to others. The LupusQoL has been validated in multiple language cohorts and countries. ${ }^{2}$ Despite its use in multiple longitudinal observational studies and two RCTs, ${ }^{2}$ the LupusQoL was not evaluated in the belimumab trials. Inclusion of SLE-specific PRO measurements in clinical trials, such as the LupusQoL, can potentially represent patients' perspectives of effects of disease and/or treatment on pertinent HRQoL domains not captured by generic metrics such as SF-36. LupusQoL includes additional pertinent domains to patients with SLE: sleep, body image, and sexual health. Two phase III RCTs, assessing the efficacy of epratuzumab in lupus, have included LupusQoL. ${ }^{59,60}$

To conclude, PRO outcomes in BLISS trials have been assessed as secondary endpoints in a responder-nonresponder analysis, which confirmed that clinical improvements measured by the SRI-4 were associated with significant and clinically meaningful reported improvements in HRQoL and fatigue. Thus, it is very important to include PROs, including generic and disease-specific measures, such as SF-36, LupusQoL, and FACIT-F, as secondary endpoints in trials assessing the efficacy of new drugs in lupus. RCTs in SLE have mainly utilized PROs to assess HRQoL, fatigue, and pain; however, as the field rapidly evolves and more trials develop, other PROs can be included to address cognitive function, anxiety, and depression, among other domains.

\section{Disclosure}

Dr Z Touma holds a Young Investigator Salary and Operating Awards provided by the Arthritis Society and the Physicians' Services Incorporated Foundation. Dr Z Touma's research is supported by a CRA (CIORA)-Arthritis Society Clinician Investigator Award. The University of Toronto Lupus Clinic is supported the Lupus Foundation of Ontario, the Lou Rocca Family, and the Kaiser's Family. Dr Z Touma has consulted for GlaxoSmithKline, Merck Serono, Pfizer, and Janssen Pharmaceuticals. Dr V Strand serves as a consultant to Human Genome Sciences, now GSK, as well as Abbvie Amgen Corporation, AstraZeneca, BMS, Boehringer Ingelheim, EMD Serono, Genentech/Roche, Glenmark, Janssen, Kypha, Lilly, Novartis, and UCB. The authors have no other relevant affiliations or financial involvement with any organization or entity with a financial interest in or financial conflict with the subject matter or materials discussed in the manuscript apart from those disclosed.

\section{References}

1. Mahieu M, Yount S, Ramsey-Goldman R. Patient-reported outcomes in systemic lupus erythematosus. Rheum Dis Clin North Am. 2016;42(2):253-263.

2. Kwan A, Strand V, Touma Z. The role of patient reported outcomes in systemic lupus erythematosus. Curr Treat Options Neurol. 2017;3(4): 308-321.

3. Strand V, Gladman D, Isenberg D, Petri M, Smolen J, Tugwell P. Outcome measures to be used in clinical trials in systemic lupus erythematosus. J Rheumatol. 1999;26(2):490-497.

4. Guidance for Industry: Systemic Lupus Erythematosus - Developing Medical Products for Treatment. 2010. Available from: https://www.fda. gov/downloads/Drugs/GuidanceComplianceRegulatoryInformation/ Guidances/ucm072063.pdf. Accessed December 12, 2018.

5. Mosca M, Costenbader KH, Johnson SR, et al. How do patients with newly diagnosed systemic lupus erythematosus present? A multicenter cohort of early systemic lupus erythematosus to inform the development of new classification criteria. Arthritis Rheumatol. Epub 2018 Jul 23.

6. Parks CG, de Souza Espindola Santos A, Barbhaiya M, Costenbader $\mathrm{KH}$. Understanding the role of environmental factors in the development of systemic lupus erythematosus. Best Pract Res Clin Rheumatol. 2017;31(3):306-320.

7. Kaul A, Gordon C, Crow MK, et al. Systemic lupus erythematosus. Nat Rev Dis Primers. 2016;2:16039.

8. Wahren-Herlenius M, Dörner T. Immunopathogenic mechanisms of systemic autoimmune disease. Lancet. 2013;382(9894):819-831.

9. Barturen G, Alarcon-Riquelme ME. SLE redefined on the basis of molecular pathways. Best Pract Res Clin Rheumatol. 2017;31(3):291-305.

10. Dörner T, Lipsky PE. Beyond pan-B-cell-directed therapy - new avenues and insights into the pathogenesis of SLE. Nat Rev Rheumatol. 2016;12(11):645-657.

11. Touma Z, Gladman DD. Current and future therapies for SLE: obstacles and recommendations for the development of novel treatments. Lupus Sci Med. 2017;4(1):e000239.

12. Al Rayes H, Touma Z. Profile of epratuzumab and its potential in the treatment of systemic lupus erythematosus. Drug Des Devel Ther. 2014;8:2303-2310.

13. Blair HA, Duggan ST. Belimumab: a review in systemic lupus erythematosus. Drugs. 2018;78(3):355-366. 
14. Crow MK, Niewold TB, Kirou KA. Cytokines and interferons in lupus. In: Wallace DJ, Hahn BH, editors. Dubois'Lupus Erythematosus and Related Syndromes. 8th ed. Philadelphia, PA: Elsevier/Saunders; 2013:62-75.

15. Stohl W, Metyas S, Tan SM, et al. B lymphocyte stimulator overexpression in patients with systemic lupus erythematosus: longitudinal observations. Arthritis Rheum. 2003;48(12):3475-3486.

16. Stohl W. Inhibition of B cell activating factor (BAFF) in the management of systemic lupus erythematosus (SLE). Expert Rev Clin Immunol. 2017;13(6):623-633.

17. Jacobi AM, Huang W, Wang T, et al. Effect of long-term belimumab treatment on B cells in systemic lupus erythematosus: extension of a phase II, double-blind, placebo-controlled, dose-ranging study. Arthritis Rheum. 2010;62(1):201-210.

18. GSK receives European marketing authorisation for self-injectable formulation of Benlysta for the treatment of systemic lupus erythematosus. Available from: https://www.gsk.com/en-gb/media/press-releases/gskreceives-european-marketing-authorisation-for-self-injectable-formulation-of-benlysta-for-the-treatment-of-systemic-lupus-erythematosus/. Accessed December 20, 2018.

19. Elalouf O, Keeling SO, Touma Z. Subcutaneous belimumab in the treatment of systemic lupus erythematosus. Immunotherapy. 2018;10(13):1163-1173.

20. Navarra SV, Guzmán RM, Gallacher AE, et al; BLISS-52 Study Group. Efficacy and safety of belimumab in patients with active systemic lupus erythematosus: a randomised, placebo-controlled, phase 3 trial. Lancet. 2011;377(9767):721-731.

21. Furie R, Petri M, Zamani O, et al; BLISS-76 Study Group. A phase III, randomized, placebo-controlled study of belimumab, a monoclonal antibody that inhibits B lymphocyte stimulator, in patients with systemic lupus erythematosus. Arthritis Rheum. 2011;63(12):3918-3930.

22. Zhang F, Bae SC, Bass D, et al. A pivotal phase III, randomised, placebo-controlled study of belimumab in patients with systemic lupus erythematosus located in China, Japan and South Korea. Ann Rheum Dis. 2018;77(3):355-363.

23. Stohl W, Schwarting A, Okada M, et al. Efficacy and safety of subcutaneous belimumab in systemic lupus erythematosus: a fifty-two-week randomized, double-blind, placebo-controlled study. Arthrits Rheumatol. 2017;69(5):1016-1027.

24. Wang C, Mayo NE, Fortin PR. The relationship between health related quality of life and disease activity and damage in systemic lupus erythematosus. $J$ Rheumatol. 2001;28(3):525-532.

25. Touma Z, Sayani A, Pineau CA, et al. Belimumab use, clinical outcomes and glucocorticoid reduction in patients with systemic lupus erythematosus receiving belimumab in clinical practice settings: results from the observe Canada Study. Rheumatol Int. 2017;37(6):865-873.

26. Collins CE, Dall'Era M, Kan H, et al. Response to belimumab among patients with systemic lupus erythematosus in clinical practice settings: 24-month results from the observe study in the USA. Lupus Sci Med. 2016;3(1):e000118.

27. Kim SS, Kirou KA, Erkan D. Belimumab in systemic lupus erythematosus: an update for clinicians. Therap Adv Chron Dis. 2012;3(1):11-23.

28. van Vollenhoven RF, Petri MA, Cervera R, et al. Belimumab in the treatment of systemic lupus erythematosus: high disease activity predictors of response. Ann Rheum Dis. 2012;71(8):1343-1349.

29. Ginzler EM, Wallace DJ, Merrill JT, et al; LBSL02/99 Study Group. Disease control and safety of belimumab plus standard therapy over 7 years in patients with systemic lupus erythematosus. $J$ Rheumatol. 2014;41(2):300-309.

30. Strand V, Levy RA, Cervera R, et al; BLISS-52 and -76 Study Groups. Improvements in health-related quality of life with belimumab, a B-lymphocyte stimulator-specific inhibitor, in patients with autoantibody-positive systemic lupus erythematosus from the randomised controlled BLISS trials. Ann Rheum Dis. 2014;73(5):838-844.

31. Furie R, Petri MA, Strand V, Gladman DD, Zhong ZJ, Freimuth WW; BLISS-52 and BLISS-76 Study Groups. Clinical, laboratory and health-related quality of life correlates of Systemic lupus erythematosus responder index response: a post hoc analysis of the phase 3 belimumab trials. Lupus Sci Med. 2014;1(1):e000031.
32. Holloway L, Humphrey L, Heron L, et al. Patient-reported outcome measures for systemic lupus erythematosus clinical trials: a review of content validity, face validity and psychometric performance. Health Qual Life Outcomes. 2014;12:116.

33. Ware JE, Sherbourne CD. The MOS 36-item short-form health survey (SF-36). I. Conceptual framework and item selection. Med Care. 1992;30(6):473-483.

34. Kiani AN, Strand V, Fang H, Jaranilla J, Petri M. Predictors of selfreported health-related quality of life in systemic lupus erythematosus. Rheumatology (Oxford). 2013;52(9):1651-1657.

35. Mok CC, Ho LY, Cheung MY, Yu KL, To CH. Effect of disease activity and damage on quality of life in patients with systemic lupus erythematosus: a 2-year prospective study. Scand J Rheumatol. 2009;38(2):121-127.

36. Urowitz M, Gladman DD, Ibanez D, et al. Changes in quality of life in the first 5 years of disease in a multicenter cohort of patients with systemic lupus erythematosus. Arthritis Care Res (Hoboken). 2014;66(9):1374-1379.

37. Rai SK, Yazdany J, Fortin PR, Avina-Zubieta JA. Approaches for estimating minimal clinically important differences in systemic lupus erythematosus. Arthritis Res Ther. 2015;17:143.

38. Annapureddy N, Devilliers H, Jolly M. Patient-reported outcomes in lupus clinical trials with biologics. Lupus. 2016;25(10):1111-1121.

39. Thumboo J, Feng PH, Boey ML, Soh CH, Thio S, Fong KY. Validation of the Chinese SF-36 for quality of life assessment in patients with systemic lupus erythematosus. Lupus. 2000;9(9):708-712.

40. Thumboo J, Fong KY, Ng TP, Leong KH, Feng PH, Thio ST, Boey ML. Validation of the MOS SF-36 for quality of life assessment of patients with systemic lupus erythematosus in Singapore. J Rheumatol. 1999;26(1):97-102.

41. Baba S, Katsumata Y, Okamoto Y, Kawaguchi Y, Hanaoka M, Kawasumi H, Yamanaka H. Reliability of the SF-36 in Japanese patients with systemic lupus erythematosus and its associations with disease activity and damage: a two-consecutive year prospective study. Lupus. 2018;27(3):407-416.

42. Nantes SG, Strand V, Su J, Touma Z. Comparison of the sensitivity to change of the 36-item short form health survey and the lupus quality of life measure using various definitions of minimum clinically important differences in patients with active systemic lupus erythematosus. Arthritis Care Res (Hoboken). 2018;70(1):125-133.

43. Touma Z, Gladman DD, Ibañez D, Urowitz MB. Is there an advantage over SF-36 with a quality of life measure that is specific to systemic lupus erythematosus? J Rheumatol. 2011;38(9):1898-1905.

44. Yilmaz-Oner S, Oner C, Dogukan FM, et al. Health-related quality of life assessed by LupusQoL questionnaire and SF-36 in Turkish patients with systemic lupus erythematosus. Clin Rheumatol. 2016;35(3):617-622.

45. McElhone K, Abbott J, Sutton C, et al. Sensitivity to change and minimal important differences of the LupusQoL in patients with systemic lupus erythematosus. Arthritis Care Res (Hoboken). 2016;68(10):1505-1513.

46. Garcia-Carrasco M, Mendoza-Pinto C, Cardiel MH, et al. Health related quality of life in Mexican women with systemic lupus erythematosus: a descriptive study using SF-36 and LupusQoL(C). Lupus. 2012;21(11):1219-1224.

47. Devilliers H, Amoura Z, Besancenot JF, et al. Responsiveness of the 36-item short form health survey and the lupus quality of life questionnaire in SLE. Rheumatology (Oxford). 2015;54(5):940-949.

48. Engel L, Beaton DE, Touma Z. Minimal clinically important difference: a review of outcome measure score interpretation. Rheum Dis Clin North Am. 2018;44(2):177-188.

49. Strand V, Crawford B. Improvement in health-related quality of life in patients with SLE following sustained reductions in anti-dsDNA antibodies. Expert Rev Pharmacoecon Outcomes Res. 2005;5(3):317-326.

50. Strand V, Aranow C, Cardiel MH, et al; LJP 394 Investigator Consortium. Improvement in health-related quality of life in systemic lupus erythematosus patients enrolled in a randomized clinical trial comparing LJP 394 treatment with placebo. Lupus. 2003;12(9):677-686. 
51. Goligher EC, Pouchot J, Brant R, et al. Minimal clinically important difference for 7 measures of fatigue in patients with systemic lupus erythematosus. J Rheumatol. 2008;35(4):635-642.

52. Cella D. Manual of the Functional Assessment of Chronic Illness Therapy (FACIT) Measurement System. Evanston, IL: Center on Outcomes, Research and Education (CORE), Evanston Northwestern Healthcare and Northwestern University; 1997.

53. Yellen SB, Cella DF, Webster K, Blendowski C, Kaplan E. Measuring fatigue and other anemia-related symptoms with the Functional Assessment of Cancer Therapy (FACT) measurement system. J Pain Symptom Manage. 1997;13(2):63-74.

54. Kosinski M, Gajria K, Fernandes AW, Cella D. Qualitative validation of the FACIT-fatigue scale in systemic lupus erythematosus. Lupus. 2013;22(5):422-430.

55. Lai JS, Beaumont JL, Ogale S, Brunetta P, Cella D. Validation of the functional assessment of chronic illness therapy-fatigue scale in patients with moderately to severely active systemic lupus erythematosus, participating in a clinical trial. J Rheumatol. 2011;38(4):672-679.
56. Strand V, Chu AD. Generic versus disease-specific measures of healthrelated quality of life in systemic lupus erythematosus. J Rheumatol. 2011;38(9):1821-1823.

57. Thumboo J, Strand V. Health-related quality of life in patients with systemic lupus erythematosus: an update. Ann Acad Med Singapore. 2007;36(2):115-122.

58. Izadi Z, Gandrup J, Katz PP, Yazdany J. Patient-reported outcome measures for use in clinical trials of SLE: a review. Lupus Sci Med. 2018;5(1):e000279.

59. Clowse ME, Wallace DJ, Furie RA, et al; EMBODY Investigator Group. Efficacy and safety of epratuzumab in moderately to severely active systemic lupus erythematosus: results from two phase III randomized, double-blind, placebo-controlled trials. Arthritis Rheumatol. 2017;69(2):362-375.

60. Fiechtner JJ, Montroy T. Treatment of moderately to severely active systemic lupus erythematosus with adrenocorticotropic hormone: a single-site, open-label trial. Lupus. 2014;23(9):905-912.
Patient Related Outcome Measures

\section{Publish your work in this journal}

Patient Related Outcome Measures is an international, peer-reviewed, open access journal focusing on treatment outcomes specifically relevant to patients. All aspects of patient care are addressed within the journal and practitioners from all disciplines are invited to submit their work as well as healthcare researchers and patient support groups.

\section{Dovepress}

The journal is included in PubMed. The manuscript management system is completely online and includes a very quick and fair peer-review system. Visit http://www.dovepress.com/testimonials.php to read real quotes from published authors. 